\title{
ENSAIOS DE SOLUBILIDADE DA UREIA EM ETANOL+METANOL E ÁGUA +METANOL
}

\author{
A.P. SILVA ${ }^{1}$, R.A.F. ANDRADE ${ }^{1}$, R.A. MALAGONI ${ }^{1}$ \\ ${ }^{1}$ Universidade Federal de Uberlândia / Faculdade de Engenharia Química \\ E-mail para contato: malagoni@feq.ufu
}

\begin{abstract}
RESUMO - O comportamento da ureia em solução é um assunto importante em estudos biológicos e ambientais. A alta solubilidade da ureia demonstrou, quando utilizada como única fonte de nitrogênio, uma tendência de ser mais eficiente que o sulfato de amônio e outras combinações de fertilizantes nitrogenados utilizados na fertirrigação. Desta forma, faz-se necessário a ampla aplicação dos estudos relacionados à solubilidade da ureia no setor industrial. Neste trabalho, o objetivo foi determinar a solubilidade da ureia em uma mistura 50\% etanol-50\% metanol e 50\% água-50\% metanol. Uma célula de equilíbrio encamisada foi utilizada nos ensaios, sendo o soluto colocado em excesso na solução. O meio foi agitado e deixado em repouso por um período de 2 horas. O sistema foi operado em temperaturas de 278,15 a $323,15 \mathrm{~K}$. Os resultados encontrados foram ajustados em função da temperatura e estão em conformidade com dados encontrados na literatura.
\end{abstract}

\section{INTRODUÇÃO}

Muitos processos que envolvem nucleação e cristalização não são totalmente compreendidos (LEE, LAHTI, 1972). Por esse motivo alguns setores da indústria química deixam de utilizar a cristalização como um método de produção, purificação ou de recuperação de materiais sólidos (LEE, LAHTI, 1972). Soluções aquosas concentradas de ureia promovem a solubilização de hidrocarbonetos. Além disso, essas soluções podem reversivelmente desnaturar a maioria das proteínas e alguns ácidos nucléicos (ISHIDA et al., 2004). Em lavouras, a fabricação de ureia permite o aumento da produção de alimentos. A ureia também é usada na alimentação do gado, como estabilizador de explosivos, na produção de resinas, de polímeros e de medicamentos.

O comportamento da ureia em solução é um assunto muito importante em estudos biológicos e ambientais, devido ao seu envolvimento como um produto residual em nossa vida diária. As interações de solutos pouco solúveis em soluções aquosas de ureia são de interesse contemporâneo, tanto a nível teórico e prático (KUMARAN et al., 2006).

Solubilidade, para SHRIVER (2003), é a propriedade de uma substância se dissolver em outra. A solubilidade é medida pela quantidade de soluto que se dissolve em uma determinada quantidade de solvente produzindo uma solução saturada, isto é, que não permite a dissolução de mais soluto. Dados de solubilidade de compostos químicos em água e em misturas de solventes são fundamentais no projeto de equipamentos industriais de separação, como: cristalizadores, extratores, evaporadores, lixiviadores e unidade de absorção. A solubilidade em água de compostos orgânicos é um dado importante em indústrias químicas, farmacêuticas, alimentícias e em aplicações ambientais (RAN et al., 2002). Os dados de solubilidade podem ser obtidos tanto 
através da realização de cálculos quanto por meio de medições. A predição de dados de solubilidade é restringida pela disponibilidade de dados termodinâmicos de componentes puros e valores de coeficientes de atividade (SAPOUNDJIEV et al., 2005). A falta de dados experimentais de solubilidade de solutos em solventes limita os estudos de desenvolvimento de modelos preditivos (BERNARDES et al., 2004)

\section{MATERIAIS E MÉTODOS}

\subsection{Unidade Experimental}

Para a realização dos experimentos foram utilizados ureia com pureza de $99 \%$ (Fabricante Labsynth), álcool etílico absoluto P.A. com pureza de 99,8\% (Vetec), álcool metílico absoluto P.A. com pureza de 99,5\% (Vetec) e água bidestilada e deionizada (Marte, DM-50). Utilizaram-se duas células de equilíbrio encamisadas fabricadas de vidro borossilicato, com entrada e saída proveniente de um banho termostatizado. A unidade experimental utilizada para determinação dos dados de solubilidade consistiu em duas células de equilíbrio ligadas em série. O banho termostatizado foi conectado na entrada da célula 1 e na saída da célula 2 . As duas células estavam ligadas entre si por uma mangueira de látex, conforme é mostrado na Figura 1. Na parte superior de cada célula foi acoplado um termopar, o qual monitorava a temperatura no centro das mesmas, e onde foram retiradas as amostras posteriormente. Cada célula foi apoiada sobre um agitador magnético que através de uma barra magnética promoveu a agitação da mistura soluto + mistura de solventes.

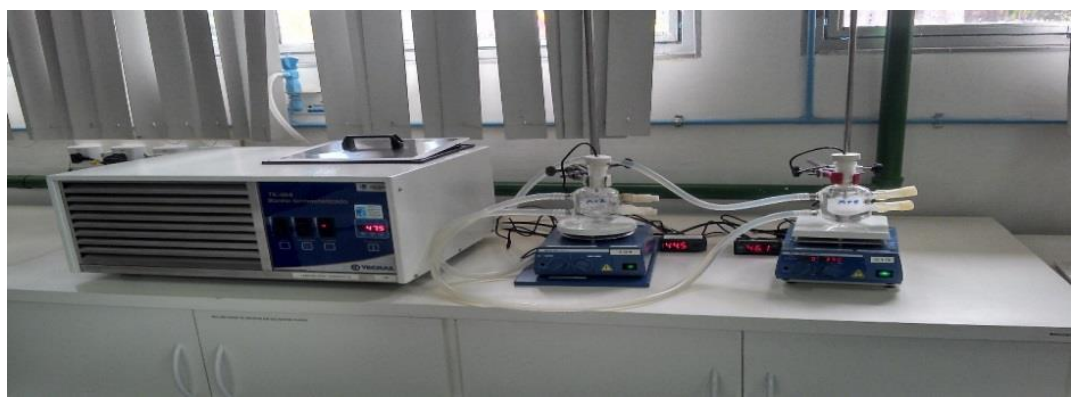

Figura 1 - Unidade Experimental

\subsection{Procedimento Experimental}

A solubilidade da ureia foi determinada em uma faixa de temperatura de 278,15 a $328,15 \mathrm{~K}$, em misturas de metanol + etanol e metanol + água com concentrações de $50 \%$ de metanol $(\mathrm{m} / \mathrm{m})$ em ambas as misturas. As soluções foram preparadas previamente em um balão volumétrico de $250 \mathrm{~mL}$; os solventes foram pesados separadamente e depois colocados no balão e agitados para homogeneizar a solução.

Duas células de equilíbrio de vidro borossilicato com capacidade de $40 \mathrm{~mL}$, encamisadas, com pontos de amostragens na lateral, foram utilizadas neste trabalho. A ureia foi adicionada em excesso na solução, a qual foi agitada por 2 horas através de agitadores magnéticos (IKA RH$\mathrm{KT} / \mathrm{C}$ ). Posteriormente, o sistema permaneceu em equilíbrio por 2 horas, mantendo-se o controle de temperatura da célula através de um banho termostatizado (Tecnal, TE-184), com precisão de $0,1^{\circ} \mathrm{C}$. Dois termopares Full Gauge (TIC-17RGTi) com precisão $0,1^{\circ} \mathrm{C}$ foram usados para 
monitorar a temperatura no interior das células. Para cada temperatura estudada, quatro amostragens de 4 a $5 \mathrm{~mL}$ foram retiradas usando uma seringa de vidro de $10 \mathrm{~mL}$, para verificação da reprodutibilidade do procedimento. As amostras foram quantificadas por gravimetria, permanecendo $24 \mathrm{~h}$ em estufa (Marconi, MA-033, precisão de $0,1^{\circ} \mathrm{C}$ ) a $338,15 \mathrm{~K}$. Após o período de secagem, as amostras contendo ureia cristalizada foram colocadas em um dessecador com sílica por $30 \mathrm{~min}$, a seguir, a massa de ureia seca foi quantificada, usando uma balança analítica (Gehaka, AY-220) com precisão de 0,0001g, para determinar o valor de solubilidade. Para cada temperatura foi feita a média aritmética dos valores de solubilidade encontrados nas amostras, para isto utilizou-se a Equação 1.

$$
S=\frac{m_{\text {ureia seca }}}{m_{\text {solvente }}} x 100
$$

Sendo, $m_{\text {ureia seca }}$ a massa da ureia seca expressa em gramas e $m_{\text {solvente, a massa do }}$ solvente também expressa em gramas.

Antes de retirar as amostras das células de equilíbrio, pesou-se os béqueres secos e limpos. Em seguida, as amostras foram retiradas, pesadas e levadas para a estufa para secagem por $24 \mathrm{~h}$ a $338,15 \mathrm{~K}$. Após a etapa de secagem, pesaram-se novamente os béqueres, que continham apenas a ureia cristalizada, as amostras foram pesadas por cerca de quatro dias até massa constante. Com os dados coletados foi possível determinar a massa de ureia cristalizada e a massa de solvente presente em cada amostra. Este procedimento foi realizado para cada faixa de temperatura. Com isto, determinou-se a solubilidade do composto em diferentes temperaturas.

\section{RESULTADOS E DISCUSSÃO}

São apresentados a seguir os dados experimentais de solubilidade da ureia em mistura de metanol+água, Tabela 1, e metanol+etanol, Tabela 2. Nessas tabelas estão reportados os desvios padrão (DP) para a temperatura e solubilidade.

A Figura 2 apresenta os dados de solubilidade da ureia em mistura de metanol + água e metanol + etanol obtidos neste trabalho, juntamente com os dados de Lee e Lahti (1972).

Ao analisar os dados experimentais de solubilidade descritos nas tabelas e na Figura 2, é possível observar que a solubilidade da ureia em ambas as misturas aumentam em função do aumento da temperatura. Nota-se também, que na mistura de metanol e água a solubilidade da ureia é maior em comparação a solubilidade na mistura de etanol e metanol, ou seja, em uma temperatura aproximada a solubilidade da ureia é menor em mistura $50 \%$ metanol $+50 \%$ etanol que em mistura contendo $50 \%$ metanol $+50 \%$ água. Isto se dá pelo fato da ureia ser mais solúvel em água que em etanol e metanol. 


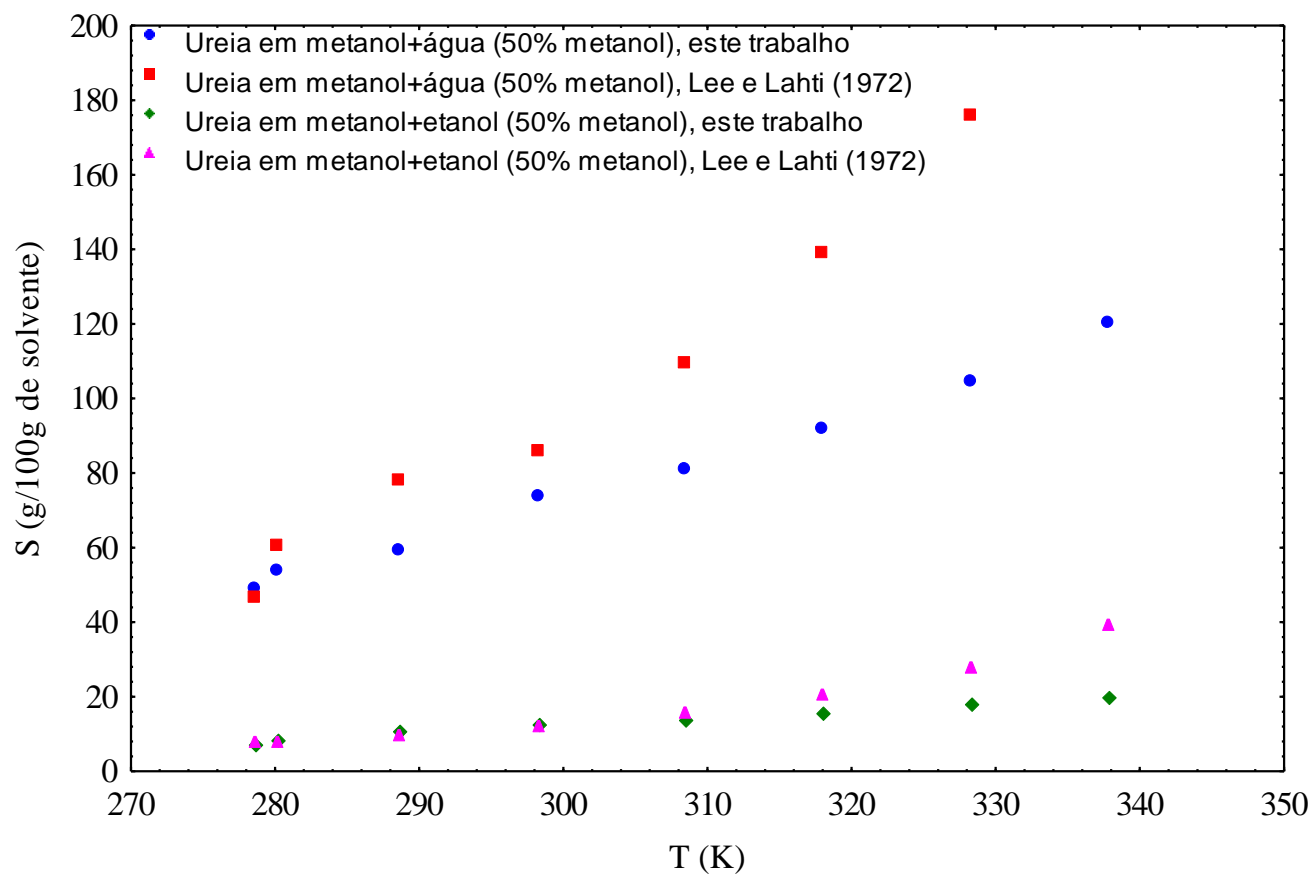

Figura 2 - Solubilidade da ureia em metanol + água e metanol + etanol

Tabela 1 - Solubilidade da ureia em metanol + água (50\% metanol m/m)

\begin{tabular}{cccc}
\hline $\boldsymbol{T} \pm \boldsymbol{\delta}(\mathbf{K})$ & $\mathbf{D P}$ & $\mathbf{S}(\mathbf{g} / \mathbf{1 0 0}$ g solvente $)$ & $\mathbf{D P}$ \\
\hline $278,3 \pm$ & 0,3 & 48,8834 & 0,18 \\
$283,2 \pm$ & 0,2 & 54,0187 & 1,65 \\
$288,1 \pm$ & 0,1 & 59,5194 & 1,85 \\
$293,3 \pm$ & 0,8 & 73,9928 & 2,13 \\
$298,4 \pm$ & 0,5 & 80,9314 & 1,6 \\
$303,4 \pm$ & 0,3 & 92,0737 & 1,37 \\
$308,3 \pm$ & 0,3 & 104,5691 & 1,73 \\
$313,1 \pm$ & 0,1 & 120,1162 & 1,35 \\
$318,3 \pm$ & 0,4 & 134,3027 & 5,2 \\
$322,8 \pm$ & 1,1 & 146,9806 & 9,74 \\
$328,2 \pm$ & 0,1 & 174,5527 & 2,92 \\
\hline
\end{tabular}


Tabela 2 - Solubilidade da ureia em metanol + etanol $(50 \%$ metanol $\mathrm{m} / \mathrm{m})$

\begin{tabular}{cccc}
\hline $\boldsymbol{T} \pm \boldsymbol{\delta}(\mathbf{K})$ & $\mathbf{D P}$ & $\mathbf{S}(\mathbf{g} / \mathbf{1 0 0} \mathbf{g}$ solvente $)$ & $\mathbf{D P}$ \\
\hline $278,1 \pm$ & 0,4 & 7,5894 & 1,91 \\
$283,1 \pm$ & 0,3 & 8,547 & 0,01 \\
$288,6 \pm$ & 0,2 & 10,846 & 0,18 \\
$293,7 \pm$ & 0,1 & 12,7843 & 0,8 \\
$298,3 \pm$ & 0,4 & 13,903 & 0,82 \\
$303,4 \pm$ & 0,2 & 15,9042 & 0,62 \\
$308,6 \pm$ & 0,1 & 17,8881 & 0,32 \\
$313,8 \pm$ & 0,1 & 20,1576 & 0,53 \\
$318,1 \pm$ & 0,2 & 21,15995 & 0,12 \\
$323,1 \pm$ & 0,3 & 27,12494 & 1,56 \\
$328,4 \pm$ & 0,1 & 29,32036 & 0,32 \\
\hline
\end{tabular}

A fim de correlacionar os dados de solubilidade em função da temperatura foram utilizados duas equações empíricas. As Equações de 2 e 3 apresentam os modelos de Lee e Lahti (1972) e Yaws et al. (1993), respectivamente.

$$
\ln S=A+B T
$$

$\log S=A^{\prime}+\frac{B^{\prime}}{T}+\frac{C^{\prime}}{T^{2}}$

Sendo: A, B, A', B' e C' os parâmetros das equações.

A seguir as Figuras 3 e 4 apresentam as curvas obtidas com os dois modelos utilizados e os dados de solubilidade na mistura metanol + água e metanol + etanol respectivamente.

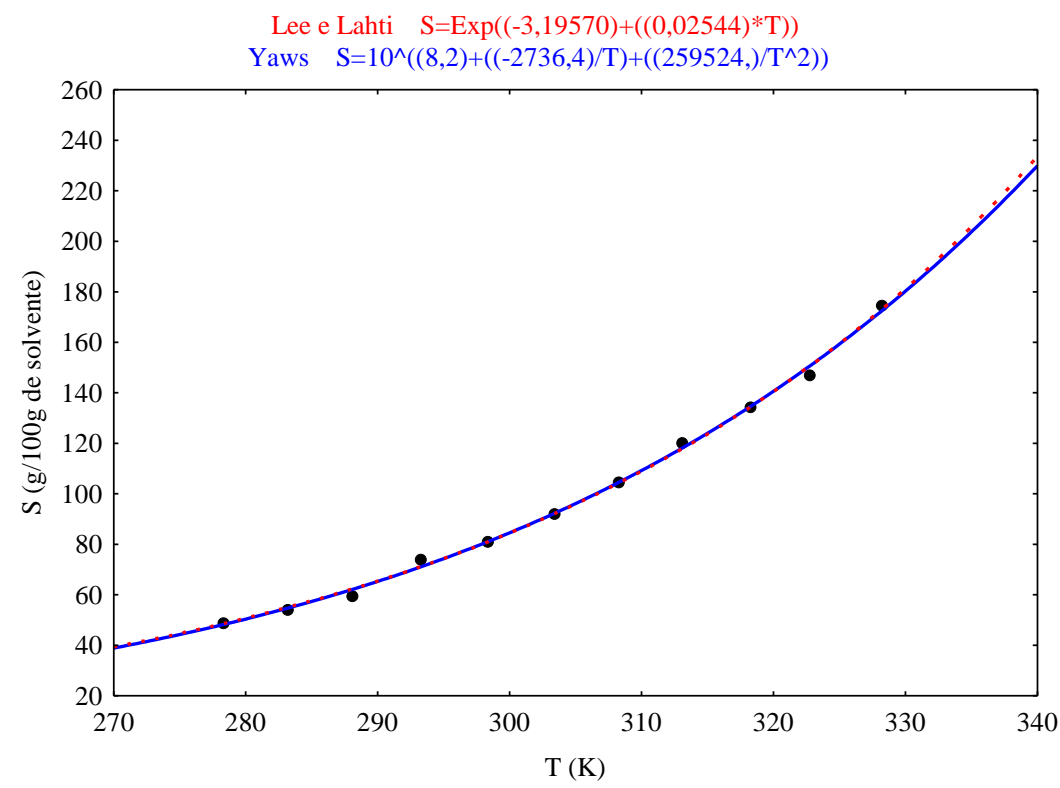

Figura 3 - Ajuste dos dados de solubilidade da ureia na mistura metanol + água 


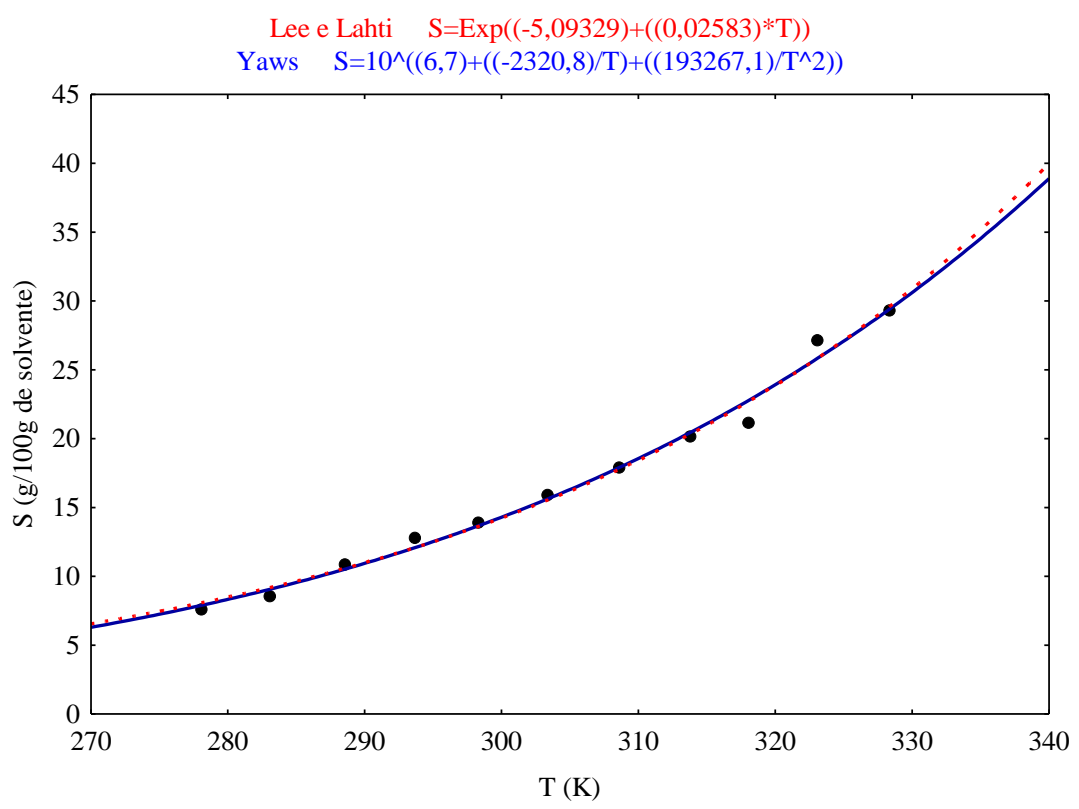

Figura 4 - Ajuste dos dados de solubilidade da ureia na mistura metanol + etanol

As Tabelas 3 e 4 reportam os valores dos parâmetros, juntamente com os desvios para cada equação empírica (Equações 2 e 3) usada no ajuste dos dados de solubilidade da ureia em mistura de metanol + água e metanol + etanol, respectivamente.

Tabela 3 - Parâmetros dos ajustes de solubilidade da mistura metanol + água

\begin{tabular}{|c|c|c|c|}
\hline \multicolumn{2}{|c|}{$\begin{array}{c}\text { Equação } 2 \\
\text { Lee e Lathi, } 1972\end{array}$} & \multicolumn{2}{|c|}{$\begin{array}{c}\text { Equação } 3 \\
\text { Yaws et al., } 1993\end{array}$} \\
\hline Parâmetro & Erro Padrão & Parâmetro & Erro Padrão \\
\hline$A=-3,19570$ & 0,139456 & $\mathrm{~A}^{\prime}=18,8$ & 3 \\
\hline $\mathrm{B}=0,02544$ & 0,000443 & $\mathrm{~B}^{\prime}=-6300,8$ & 1844,4 \\
\hline- & & $C^{\prime}=597578,8$ & 282551,6 \\
\hline \multicolumn{2}{|c|}{$\mathrm{R}^{2}=0,9989$} & \multicolumn{2}{|c|}{$\mathrm{R}^{2}=0,9989$} \\
\hline
\end{tabular}

Tabela 4 - Parâmetros dos ajustes de solubilidade da mistura metanol + etanol

\begin{tabular}{|c|c|c|c|}
\hline \multicolumn{2}{|c|}{$\begin{array}{c}\text { Equação } 2 \\
\text { Lee e Lathi, } 1972 \\
\end{array}$} & \multicolumn{2}{|c|}{$\begin{array}{c}\text { Equação } 3 \\
\text { Yaws et al., } 1993 \\
\end{array}$} \\
\hline Parâmetro & Erro Padrão & Parâmetro & Erro Padrão \\
\hline$A=-5,09329$ & 0,313254 & $\mathrm{~A}^{\prime}=15,5$ & 6,7 \\
\hline $\mathrm{B}=0,02583$ & 0,000994 & $\mathrm{~B}^{\prime}=-5343,8$ & 4118,8 \\
\hline- & - & $C^{\prime}=445009,4$ & 631467,9 \\
\hline \multicolumn{2}{|c|}{$\mathrm{R}^{2}=0,9945$} & \multicolumn{2}{|c|}{$\mathrm{R}^{2}=0,9946$} \\
\hline
\end{tabular}

Analisando os gráficos e as tabelas e considerando um nível de significância de $5 \%$ no software Statistica, para a mistura de metanol e água, foi possível verificar que o parâmetro $C^{\prime}$ do modelo Yaws et al., (1993) não é significativo. Para um mesmo nível de significância, na mistura metanol e etanol, observou-se que os parâmetros $B^{\prime}$ e $C^{\prime}$ do modelo de Yaws et al., (1993) não 
são significativos, os parâmetros A e B de Lee e Lahti (1972) foram significativos para ambas misturas de solventes.

Observando os valores de $R^{2}$, valor do parâmetro, erro padrão do parâmetro, nível de significância e quantidade de parâmetros do modelo, pode-se concluir que para as duas misturas de solventes propostas (metanol + água e metanol + etanol) o modelo proposto por Lee e Lahti (1972) foi o que melhor representou os dados experimentais de solubilidade. As Tabelas 5 e 6 reportam os valores observados, preditos e os resíduos obtidos no ajuste, usando o melhor modelo, para as misturas de metanol + água e metanol + etanol, respectivamente.

Tabela 5 - Resíduos obtidos usando o modelo de Lee e Lahti (1972) na mistura metanol + água

\begin{tabular}{cccc}
\hline T $(\mathbf{K})$ & Valor Observado & Valor Predito & Resíduos \\
\hline 278,32 & 48,8834 & 48,6331 & 0,25029 \\
283,18 & 54,0187 & 55,0331 & $-1,01441$ \\
288,08 & 59,5194 & 62,3387 & $-2,81934$ \\
293,25 & 73,9928 & 71,1009 & 2,89194 \\
298,35 & 80,9314 & 80,9503 & $-0,01888$ \\
303,42 & 92,0737 & 92,0938 & $-0,02010$ \\
308,28 & 104,5691 & 104,2131 & 0,35597 \\
313,08 & 120,1162 & 117,7475 & 2,36873 \\
318,28 & 134,3027 & 134,4002 & $-0,09749$ \\
322,75 & 146,9806 & 150,5855 & $-3,60495$ \\
328,22 & 174,5527 & 173,0671 & 1,48563 \\
\hline
\end{tabular}

Tabela 6 - Resíduos obtidos usando o modelo de Lee e Lahti (1972) na mistura metanol + etanol

\begin{tabular}{cccc}
\hline $\mathbf{T}(\mathbf{K})$ & Valor Observado & Valor Predito & Resíduos \\
\hline 278,08 & 7,58940 & 8,07940 & $-0,49000$ \\
283,08 & 8,54700 & 9,19319 & $-0,64619$ \\
288,55 & 10,84600 & 10,58830 & 0,25770 \\
293,68 & 12,78430 & 12,08849 & 0,69581 \\
298,32 & 13,90300 & 13,62766 & 0,27534 \\
303,38 & 15,90420 & 15,53038 & 0,37382 \\
308,62 & 17,88810 & 17,78123 & 0,10687 \\
313,78 & 20,15760 & 20,31628 & $-0,15868$ \\
318,05 & 21,15995 & 22,68521 & $-1,52526$ \\
323,08 & 27,12494 & 25,83253 & 1,29241 \\
328,35 & 29,32036 & 29,59942 & $-0,27906$ \\
\hline
\end{tabular}

É possível notar ao analisar a Tabela 5, que os resíduos foram em média iguais a 1,3571, pela Tabela 6 verifica-se que os resíduos foram em média iguais a 0,5547, relativamente baixos, ou seja, os valores experimentais e preditos ficaram bem próximos ao utilizar o modelo proposto por Lee e Lahti (1972) para representar os resultados de solubilidade nas misturas de solvente de metanol + água e metanol + etanol. 
Mais estudos da solubilidade de ureia em outros solventes podem ser encontrados nos trabalhos de Carvalho et al. (2012), Leão et al. (2011), Maionchi et al. (2013), Fontes et al. (2013) e no trabalho de Diniz et al. (2014) apresentado também neste congresso.

\section{CONCLUSÃO}

Com a realização deste trabalho pôde-se concluir que o método gravimétrico é bastante aceitável para a determinação de solubilidade e que ao utilizar este método fica explícita a influência da temperatura no valor da solubilidade. Ao analisar os resultados, conclui-se que com o aumento da temperatura há também um aumento significativo na solubilidade da ureia em ambas as misturas de solvente. Nota-se que na mistura 50\% metanol - 50\% água o valor da solubilidade é significativamente maior que na mistura 50\% metanol - 50\% etanol. Esta afirmação pode ser verificada ao avaliar os dados experimentais deste trabalho e dados da literatura.

O modelo proposto por Lee e Lahti (1972) foi o que melhor ajustou os dados experimentais da ureia nas duas misturas de solventes, apresentando baixos valores de resíduos entre o valor observado e o valor predito.

\section{AGRADECIMENTOS}

Agradecemos a Fundação de Amparo à Pesquisa do Estado de Minas Gerais (FAPEMIG) pelos recursos concedidos no Projeto de Participação Coletiva em Eventos Técnicos-Científicos (PCE-00082-14) e a Faculdade de Engenharia Química da Universidade Federal de Uberlândia pela estrutura física disponibilizada para a realização desta pesquisa.

\section{REFERÊNCIAS}

BERNARDES, E.A.; BRUNETTO, R.S.; FRANCO JR., M.R. Coeficientes de atividade a diluição infinita aplicados ao estudo da poluição aquática. XV Congresso Brasileiro de Engenharia Química, Curitiba, 2004.

CARVAlHO, N. D. ; CASTRO, C. C. ; LOBATO, F. S. ; MALAGONI, R. A. . Solubilidade da ureia em etanol+água. In: XIX COBEQ - Congresso Brasileiro de Engenharia Química, 2012, Búzios - RJ. Anais do XIX COBEQ, 2012. p. 5421-5430.

DINIZ, V.E.; Determinação da solubilidade de ureia em misturas etanol-água em temperaturas de 278,15 a 333,15K. 2014. 75f. Trabalho de conclusão de curso. Uberlândia: Universidade Federal de Uberlândia, 2014.

FONTES, A. V.; BASSI, C. M. M. ; MAIONCHI, I. A.; MALAGONI, R. A. Solubilidade da ureia em mistura isopropanol+água. In: X COBEQ IC - Congresso Brasileiro de Engenharia Química Iniciação Científica, Vassouras - RJ, 2013. p. 1-6.

ISHIDA, T.; ROSSKY, P. J.; CASTINER, E. W. A Theoretical investigation of the shape and hydration properties of aqueous urea: evidence for nonplanar urea geometry. J. Phys.

Chem. B, 108, 17583-17590, 2004.

KUMARAN, P.; RAMAMURTHY, P. PET suppression of acridinedione dyes by urea derivatives in water and methanol. J. Phys. Chem. B, 110, 47, p. 23783-23789, 2006. 
LEÃO, A. M. ; CARVAlHO, N. D. ; LOBATO, F. S. ; MALAGONI, R. A. Determinação experimental da solubilidade de ureia em água. In: VI CBTermo - Congresso Brasileiro de Termodinâmica Aplicada, Salvador - BA, 2011. p. 1-6.

LEE, F.-M.; LAHTI, L. E. Solubility of urea in water-alcohol mixtures. J. Chem. Eng. Data, 17, 3, 304-306, 1972.

MAIONCHI, I. A. ; BASSI, C. M. M. ; CARVALHO, N. D. ; MALAGONI, R. A. Determinação da solubilidade da ureia em misturas aquosas de isopropanol usando gravimetria. In: VII CBTermo - Congresso Brasileiro de Termodinâmica Aplicada, Uberlândia - MG, 2013. p. $1-10$.

RAN, Y.; HE, Y.; YANG, G.; JOHNSON, J. L. H.; YALKOWSKY, S. H. Estimation of aqueous solubility of organics compounds by using the general solubility equation. Chemosphere, v. 48, p. 487-509, 2002.

SHRIVER, D. F.; ATKINS, P. W. Química inorgânica. $3^{\text {a }}$ edição. São Paulo. Ed. Bookman, 2003.

YAWS, C. L.; LIN, X.; BU, L. The water solubility of naphtenes. Chemical Engineering, v. 10, p. 122-123, 1993. 